\title{
Water fluoridation and hypothyroidism: results of this study need much more cautious interpretation
}

\author{
John N Newton, ${ }^{1}$ Nick Young, ${ }^{2}$ Julia Verne, ${ }^{2}$ John Morris $^{3}$
}

Dental caries remains a significant public health problem in many countries and an important cause of health inequalities. In England, almost a third of 5-year-old children and over two-fifths of 15-year-old teenagers are affected, and substantially higher rates of disease are seen among deprived communities. ${ }^{1}$ Dental disease can cause impaired nutrition and growth, ${ }^{2} 3$ and is one of the most common causes of child hospital admission. Long-term impacts on appearance, speech, schooling and self-confidence may add up to a substantial disadvantage for affected children.

Water fluoridation schemes were first introduced in England in the 1950s and around six million people across the country now live in areas where the level of fluoride in drinking water is adjusted to an optimum level for oral health. Worldwide, hundreds of millions of people have experienced the benefits of water fluoridation for many decades. The possible health effects of water fluoridation have been studied and reviewed many times. ${ }^{4}$ The dental effects of fluoridation, namely reduced dental decay and dental fluorosis, are well described. Water fluoridation is one of the few interventions that can be expected to directly reduce public health inequalities, although the empirical evidence for this remains relatively weak. Many non-dental health conditions have been alleged as a consequence of water fluoridation, but there is no consistent scientific evidence to support any of these putative associations. In general, the literature suggesting adverse health effects of fluoridation is characterised by poor-quality studies that do not adequately adjust for potential confounding variables.

We have a number of concerns about the paper published this week in $J E C H$ reporting an apparent association between

\footnotetext{
${ }^{1}$ Public Health England, London, UK; ${ }^{2}$ South West Knowledge and Intelligence Team, Public Health England, Bristol, UK; ${ }^{3}$ Dental Public Health, Public Health England, Birmingham, UK
}

Correspondence to Professor John N Newton, Public Health England, Wellington House, 133-155 Waterloo Road, London SE1 8UG, UK; john.newton@phe.gov.uk water fluoridation and hypothyroidism. ${ }^{6}$ The authors have not established a clear prior hypothesis for the association, have misrepresented the conclusions of the existing literature, seem not to have taken adequate account of the potential for confounding, have categorised variables with arbitrary cut-offs that deviate from normal practice, and seem to have made a basic error in reporting the results of their own model. Most important is that they have drawn conclusions which greatly exceed the evidence available from this study, even if the methods used had been reliable.

Hypothyroidism in this country is largely an autoimmune disease, the aetiology of which is well described. ${ }^{7}$ The evidence provided in support of the authors' prior hypothesis of an association with water fluoridation is, therefore, likely to be irrelevant to patients listed on Quality and Outcomes Framework registers in England in 2012, who are not generally at risk of iodine deficiency. In addition, some $20-30 \%$ of these patients will be receiving levothyroxine because of previous thyroid ablation by surgery or radiation therapy to treat byperthyroidism, ${ }^{7}$ which is even less likely to be in any way related to fluoride ingestion.

As Grimes has already pointed out in another commentary on this article, ${ }^{8}$ the authors mistakenly imply that a connection between water fluoridation and hypothyroidism is established in the literature. The weakness of any such prior hypothesis is important because ecological studies, such as this one, are highly susceptible to spurious associations as a result of confounding ${ }^{9}$ and subsequent publication bias in favour of positive results. ${ }^{10}$ Hypothyroidism is very strongly associated with age and sex; therefore, there is considerable scope for inadequately controlled confounding by these or other factors explaining the level of association observed in this study. There is no mention of potential confounding in the paper.

In relation to the methods used, we also have significant concerns. The authors provide no justification for the way that various data sets were reduced into categorical variables, for example, two of the tertiles of deprivation were inexplicably combined; likewise, the upper tertile of hypothyroidism prevalence was used instead of actual prevalence. This approach can only reduce the performance of their model. In the same way, we see no justification for selecting two areas of the country for analysis when data are available for the whole country. It also matters how those areas are defined as there are a number of options, the choice of which would affect the results.

Of most obvious concern is the reported result on relationship between deprivation and hypothyroidism. Peckham et al state, using their final multivariable model, 'the odds of a practice reporting high levels of hypothyroidism is 1.7 times higher where the IMD [Index of Multiple Deprivation] is medium or high'. Surprised to see a positive relationship, we analysed the data ourselves and found that the univariate odds of a practice reporting high levels of hypothyroidism (as defined in the paper) were less (OR 0.49 ) when a practice was in the upper two tertiles of IMD score compared to the least deprived tertile. In other words, the relationship was in the other direction. We cannot see an obvious explanation for this difference and it looks like an error.

The biggest problem with this paper, however, is in the interpretation which puts far too much weight on such weak evidence. The approach used is notoriously unreliable as a way of identifying independent associations and the lack of a clearly established prior hypothesis make it very unconvincing as evidence of a causal relationship. Given the other problems we have identified, this loose interpretation is a very serious concern. Such speculation is likely to result in unfounded public anxiety about a public health intervention which currently protects the health of children's teeth in many parts of the world.

\section{Competing interests None declared.}

Provenance and peer review Not commissioned; internally peer reviewed.
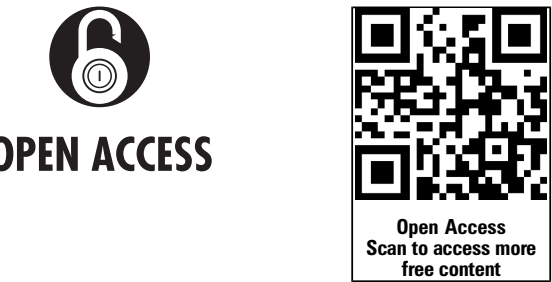
Open Access This is an Open Access article distributed in accordance with the Creative Commons Attribution Non Commercial (CC BY-NC 4.0) license, which permits others to distribute, remix, adapt, build upon this work non-commercially, and license their derivative works on different terms, provided the original work is properly cited and the use is noncommercial. See: http://creativecommons.org/licenses/ by-nc/4.0/

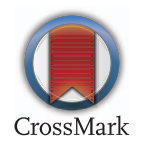

To cite Newton JN, Young N, Verne J, et al. J Epidemiol Community Health 2015;69:617-618.

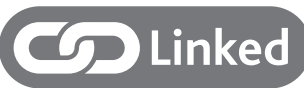

- http://dx.doi.org/10.1136/ jech-2015-205708

- http://dx.doi.org/10.1136/ jech-2014-204971
J Epidemiol Community Health 2015;69:617-618. doi:10.1136/jech-2015-205917

\section{REFERENCES}

1 Child Dental Health Survey 2013, England, Wales and Northern Ireland (NS): Health and Social Care Information Centre. 2015. http://www.hscic.gov.uk/ catalogue/PUB17137 (accessed 13 Apr 2015)

2 Shepherd MA, Nadanovsky P, Sheiham A. The prevalence and impact of dental pain in 8-year-old school children in Harrow, England. Br Dent J 1999;187:38-41.

3 Clarke M, Locker D, Berall G, et al. Malnourishment in a population of young children with severe early childhood caries. Pediatr Dent 2006;28: 254-9.

4 McDonagh MS, Whiting PF, Wilson PM, et al. Systematic review of water fluoridation. BMJ 2000;321:855-9.

5 Yeung CA. A systematic review of the efficacy and safety of fluoridation. Evid Based Dent 2008;9:39-43.
6 Peckham S, Lowery D, Spencer S. Are fluoride levels in drinking water associated with hypothyroidism prevalence in England? A large observational study of GP practice data and fluoride levels in drinking water. J Epidemiol Community Health 2015;69: 619-24.

7 Vanderpump MP, Tunbridge WM. Epidemiology and prevention of clinical and subclinical hypothyroidism. Thyroid 2002;12:839-47.

8 Grimes DR. Commentary on Are fluoride levels in drinking water associated with hypothyroidism prevalence in England? A large observational study of GP practice data and fluoride levels in drinking water. J Epidemiol Community Health 2015;69:616.

9 Greenland S, Morgenstern H. Ecological bias, confounding, and effect modification. Int J Epidemiol 1989;18:269-74.

10 Easterbrook PJ, Berlin JA, Gopalan R, et al. Publication bias in clinical research. Lancet 1991;337:867-72. 